\title{
Coexistent of paradoxical herniation and subdural hygroma: a case report
}

\author{
Yi Feng and Yuhai Wang*
}

\begin{abstract}
Background: Coexistent of paradoxical herniation and subdural hygroma (SDG) is very rare, confusing symptoms may mislead the therapies.

Case presentation: We report a case of a 33-year-old man underwent decompressive craniectomy (DC), postoperatively, the patient developed progressive neurologic deterioration and midline shift opposite to the DC window, CT scan revealed a SDG beside the DC window, instead of attributing the midline shift to the SDG, we recognized the underlying paradoxical herniation according to the sunken skin flap and history of lumbar cistern drainage. Subsequently we treat him with intravenous fluid expansion therapy instead of draining from the SDG, the patient recovered in one day and no recurrence was found later.

Conclusion: For patients underwent DC, we should pay attention to the occurrence of paradoxical herniation, SDG following DC may be the consequence of paradoxical herniation caused by CSF reduce therapy in post-DC patient, intravenous fluid expansion therapy is recommended and would play a great role for the recovery of the patient rather than surgical management.
\end{abstract}

Keywords: Paradoxical herniation, Subdural hygroma, Decompressive craniectomy

\section{Background}

The paradoxical herniation is a rare complication happened after DC (decompressive craniectomy), it has been recognized in recent years, which presented with progressive neurologic deterioration and midline shift, and can be treated under timely intravenous infusion. This is a case with coexistent of paradoxical herniation and SDG, which is more confusing and misleading the doctors to its attributing cause of midline shift to SDG instead of paradoxical herniation.

\section{Case presentation}

A 33-year-old man initially presented to the emergency room with $24 \mathrm{~h}$ of coma after heavy drinking with a GCS (Glasgow coma score) of 6, dilated left pupil without direct and indirect light reflex. Initial CT (Computed Tomography) scan was reported as left temporal epidural hematoma, with a severe middle line shift to the right (Fig. 1).

\footnotetext{
* Correspondence: yuhaiwangcn@gmail.com

Anhui Medical University, School of Clinical medicine (WUXI 101-hospital), 101\# Xingyuan Road, Wuxi, 214000 Jiangsu, P.R.C
}

Hematoma evacuation and DC was performed immediately. The patient recovered awareness gradually in 3 days, postoperative $\mathrm{CT}$ scan revealed the hematoma was totally evacuated. 6 days post-DC, CSF leakage from the incision was identified, and continuous lumbar cistern drainage was implemented from CSF drainage, 2 days later, sudden drop of consciousness happened with left pupil distortion, CT scan revealed a left frontal region SDG beside the cranial defects, with midline-shift to the right and disappearance of ambient cistern. Surprisingly, we found the skin flap was sunken, instead of surgical drainage of SDG, we gave a rapid intravenous fluid expansion therapy, by $4000 \mathrm{ml}$ fluid including human albumin $(10 \% 20 \mathrm{~g})$ and plasma $(350 \mathrm{ml})$ in $24 \mathrm{~h}$, the flap of cranial defect went up gradually, the patient's consciousness progressed in $24 \mathrm{~h}$ to full consciousness, CT scan (Fig. 2) in the next day revealed enlargement of the sylvian fissure, ambient cistern and aqueduct of midbrain, also the middle line returned, the sub-skull SDG changed into sub-skin flap SDG and then disappeared gradually. The patient recovered rapidly and was discharged 3 days later with a minor diplopia which was
C Biomed Central

(c) 2015 Feng and Wang. Open Access This article is distributed under the terms of the Creative Commons Attribution 4.0 International License (http://creativecommons.org/licenses/by/4.0/), which permits unrestricted use, distribution, and reproduction in any medium, provided you give appropriate credit to the original author(s) and the source, provide a link to the Creative Commons license, and indicate if changes were made. The Creative Commons Public Domain Dedication waiver (http://creativecommons.org/publicdomain/zero/1.0/) applies to the data made available in this article, unless otherwise stated. 


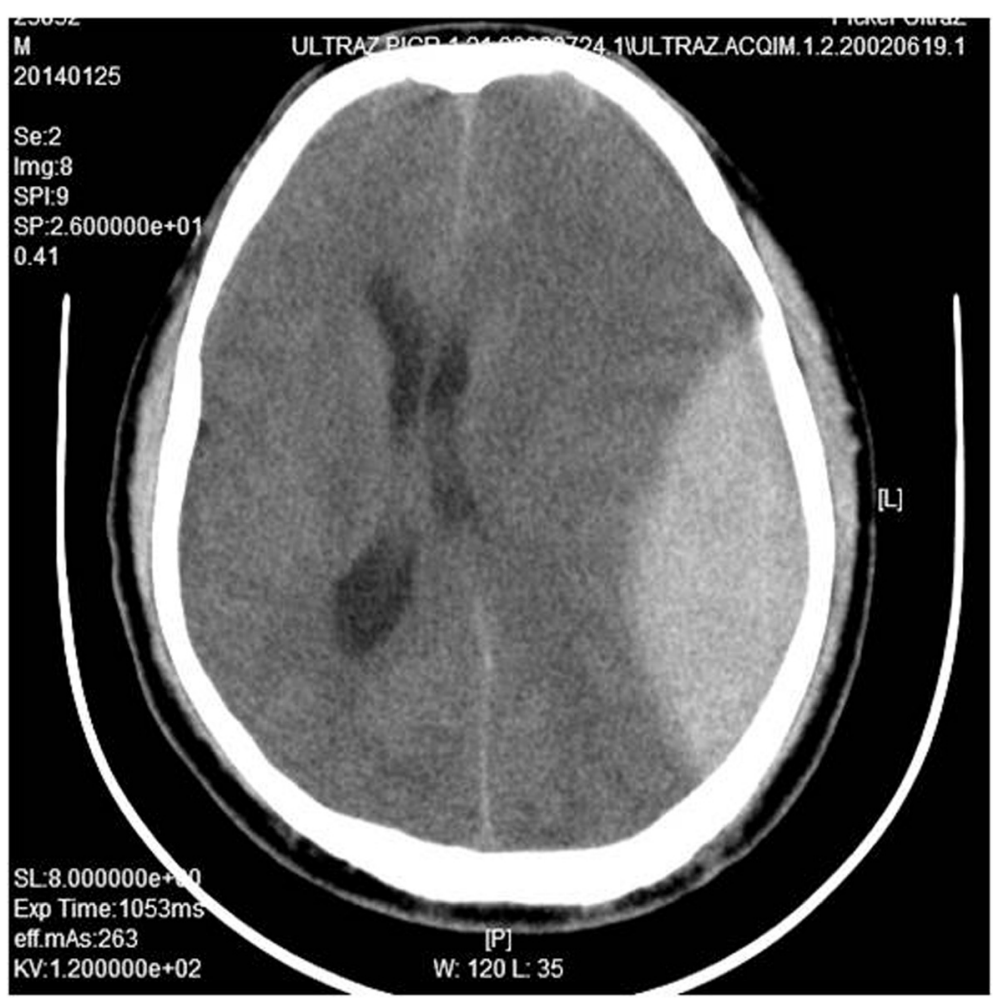

Fig. 1 Preoperative CT was reported as left temporal epidural hematoma, with a severe middle line shift to the right

recovered in 2 months. With 4 months of subsequent follow ups showed no recurrence.

\section{Discussion}

The term paradoxical herniation was first used by Schwab et al. in 1998 [1], it is a rare, potentially lifethreatening but treatable complication, with progressive neurologic deterioration, sunken skull defect, midline shift, and compression of the brainstem . It may present in a delayed fashion after a lumbar puncture in patients with DC, Vilela [2] explained the cause that with a large skull defect, a lumbar puncture exacerbates the negative pressure gradient between the atmosphere and the cranium. Large dose of mannitol administration may aggravate the situation. For treatment, patients should be treated with trendelenburg position, and clamping of CSF drainage, dehydration therapy should be discontinued instead of hyperosmolar with colloid [3, 4].

Oyelese A et al. [5] report a case of paradoxical herniation from intracranial hypotension secondary to a lumbar puncture. This patient developed a large right hemispheric stroke after suprasellar meningioma resection, immediate DC was performed to reduce intracranial hypotension. 1 month after decompressive hemicraniectomy the patient developed a large subgaleal and subdural hygroma, 4 days of LP (lumber acupuncture) was performed followed by sudden consciousness loss and bilateral pupil dilation, a huge dose of mannitol administration even worsen the situation. Fields et al. [6] report a patient 43 with a cerebral contusion and a subdural hematoma who underwent $\mathrm{DC}$ and medium pressure ventriculoperitoneal shunt. Two months later after an LP, his condition markedly deteriorated, a brain CT scan showed a mark with the midline shift in the direction opposite the craniectomy site with subfalcine herniation and effacement of the peripontine cisterns. Given the concern for paradoxical herniation, $5 \mathrm{~h}$ after his initial deterioration, he was placed in the trendelenburg position, intravenous fluids were administered, and the VPS was tied off, his condition improved within $1 \mathrm{~h}$.

SDG is a kind of CSF hydrodynamic disturbances frequently caused by DC [7-11]. The contralateral SDG were constantly reported, the aetiology was thought to combine with external cerebral herniation. This outward herniation combined with rapid reduction in ICP may incite a pressure gradient between the two hemispheres and lead to the enlargement of the contralateral subdural space and the accumulation of effusion, especially when, initially, there is a possible rupture in the arachnoid layer after head trauma [12-14]. 


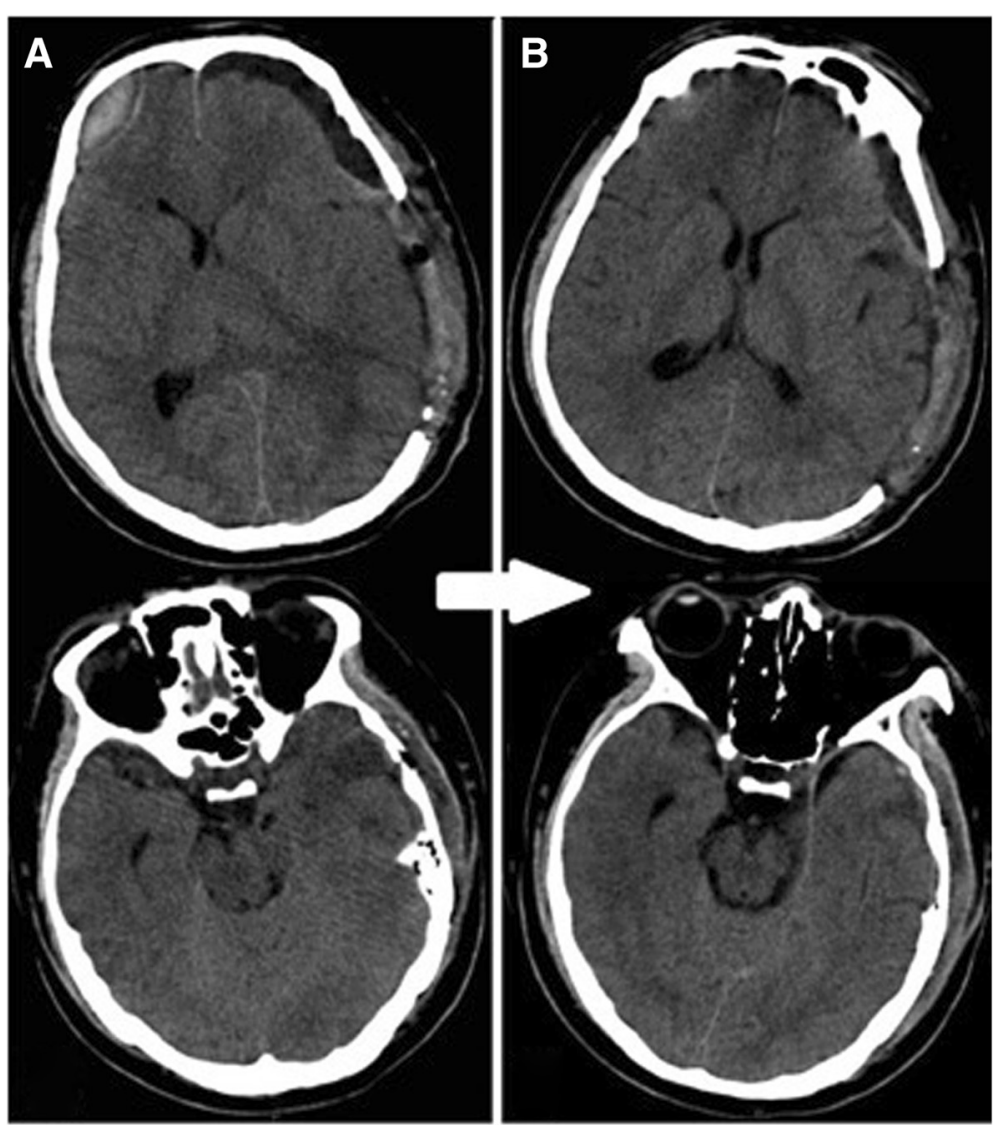

Fig. 2 a 8 day post-DC, the patient presented with progressive neurologic deterioration and a sunken skull defect, CT notified a shifted midline, left frontal SDG in the DC side, $\mathbf{b} 9$ days post-DC, after intravenous infusion, the patient recovered awareness with return of midline and enlargement of ambient cistern in $C T$

In this case, SDG harbored in the opposite side of midline shift besides the cranial defect has never been reported before. We supposed, the brain translocation caused by paradoxical herniation may be the initiating factors of this kind of SDG. Without the knowledge of paradoxical herniation, we could easily come to the wrong conclusion that the mass effect of SDG pushed the midline to the opposite site. If a borehole or lumbar acupuncture was performed to solve the SDG, the neurologic deterioration will continue because of the rising of paradoxical herniation and pressure imbalance after CSF lost [5]. Under the "do no harm" principle, patients with coexistent of paradoxical herniation and SDG without severe neurologic deterioration, could be tentatively given high dose of intravenous infusion, before we take any surgical treatment. But, for these patients with severe midline shift, the none-surgical therapy is untried and need further prove.

\section{Conclusion}

For patients underwent DC, we should pay attention to the occurrence of paradoxical herniation, high threshold should be adopted in performing lumbar puncture and the usage of mannitol. SDGs following DC may be the consequence of paradoxical herniation caused by CSF reduce therapy in post-DC patient, intravenous fluid expansion therapy is recommended and would play a great role for the recovery of the patient rather than surgical management.

\section{Consent}

Written informed consent was obtained from the patient for publication of this Case report and accompanying images. A copy of the written consent is available for review by the Editor-in-Chief of this journal.

\section{Competing interests}

The authors declare that they have no competing interests.

\section{Authors' contributions}

YF collected all the data and wrote the article. YW. guided the treatment and helped to draft the manuscript. All authors intellectually read and approved the final manuscript.

\section{Authors' information}

Not applicable 


\section{Acknowledgments}

I would like to express our gratitude to the people who made this dissertation possible. Thanks to my dissertation committee for their useful feedback and direction in conducting this study. To Mr. Likun Yang for critically reading the manuscript and to Mr. Jie Zhu, M.D. for helping us with the entire process from surgery to management of the patient. Their effort and dedication to this project is unmeasurable.

Received: 28 April 2015 Accepted: 17 September 2015

Published online: 18 December 2015

\section{References}

1. Schwab S, Erbguth F, Aschoff A, Orberk E, Spranger M, Hacke W. ["Paradoxical" herniation after decompressive trephining]. Nervenarzt. 1998;69(10):896-900.

2. Vilela MD. Delayed paradoxical herniation after a decompressive craniectomy: case report. Surg Neurol. 2008;69(3):293-6. discussion 296.

3. Stiver SI. Complications of decompressive craniectomy for traumatic brain injury. Neurosurg Focus. 2009;26(6), E7.

4. Akins PT, Guppy KH. Sinking skin flaps, paradoxical herniation, and external brain tamponade: a review of decompressive craniectomy management. Neurocrit Care. 2008;9(2):269-76.

5. Oyelese AA, Steinberg GK, Huhn SL, Wijman CA. Paradoxical cerebral herniation secondary to lumbar puncture after decompressive craniectomy for a large space-occupying hemispheric stroke: case report. Neurosurgery. 2005;57(3):E594. discussion E594.

6. Fields JD, Lansberg MG, Skirboll SL, Kurien PA, Wijman CA. "Paradoxical" transtentorial herniation due to CSF drainage in the presence of a hemicraniectomy. Neurology. 2006;67(8):1513-4.

7. Guerra WK, Gaab MR, Dietz H, Mueller JU, Piek J, Fritsch MJ. Surgical decompression for traumatic brain swelling: indications and results. Neurosurg. 1999;90(2):187-96.

8. Honeybul S, Ho KM. Incidence and risk factors for post-traumatic hydrocephalus following decompressive craniectomy for intractable intracranial hypertension and evacuation of mass lesions. J Neurotrauma. 2012;29(10):1872-8.

9. De Bonis P, Pompucci A, Mangiola A, Rigante L, Anile C. Post-traumatic hydrocephalus after decompressive craniectomy: an underestimated risk factor. J Neurotrauma. 2010;27(11):1965-70.

10. Aarabi B, Chesler D, Maulucci C, Blacklock T, Alexander M. Dynamics of subdural hygroma following decompressive craniectomy: a comparative study. Neurosurg Focus. 2009;26(6):E8.

11. Honeybul S, Ho KM. Decompressive craniectomy for severe traumatic brain injury: the relationship between surgical complications and the prediction of an unfavourable outcome. Injury. 2014.

12. Chieregato A, Fainardi E, Morselli-Labate AM, Antonelli V, Compagnone C, Targa L. Factors associated with neurological outcome and lesion progression in traumatic subarachnoid hemorrhage patients. Neurosurgery. 2005;56(4):671-80. discussion 671-680.

13. Su TM, Lee TH, Chen WF, Lee TC, Cheng CH. Contralateral acute epidural hematoma after decompressive surgery of acute subdural hematoma: clinical features and outcome. J Trauma. 2008;65(6):1298-302.

14. Yang XF, Wen L, Li G, Zhan RY, Ma L, Liu WG. Contralateral subdural effusion secondary to decompressive craniectomy performed in patients with severe traumatic brain injury: incidence, clinical presentations, treatment and outcome. Med Princ Pract. 2009:18(1):16-20.

\section{Submit your next manuscript to BioMed Central and take full advantage of:}

- Convenient online submission

- Thorough peer review

- No space constraints or color figure charges

- Immediate publication on acceptance

- Inclusion in PubMed, CAS, Scopus and Google Scholar

- Research which is freely available for redistribution 